\title{
POSITIVE SOLUTIONS OF SOME NONLOCAL BOUNDARY VALUE PROBLEMS
}

\author{
GENNARO INFANTE AND J. R. L. WEBB
}

Received 19 October 2002

We establish the existence of positive solutions of some $m$-point boundary value problems under weaker assumptions than previously employed. In particular, we do not require all the parameters occurring in the boundary conditions to be positive. Our results allow more general behaviour for the nonlinear term than being either sub- or superlinear.

\section{Introduction}

Recently, much attention has been paid to the study of certain nonlocal boundary value problems (BVPs), whose study has been motivated by the work of Bitsadze and Samarskii [1] and Il'in and Moiseev [7].

In particular, existence of solutions for the so-called $m$-point BVPs

$$
u^{\prime \prime}(t)+g(t) f(u(t))=0 \quad(0<t<1)
$$

under one of the boundary conditions (BCs)

$$
\begin{aligned}
& u^{\prime}(0)=0, \quad u(1)=\sum_{i=1}^{m-2} \alpha_{i} u\left(\eta_{i}\right), \quad 0<\eta_{1}<\eta_{2}<\cdots<\eta_{m-2}<1, \\
& u(0)=0, \quad u(1)=\sum_{i=1}^{m-2} \alpha_{i} u\left(\eta_{i}\right), \quad 0<\eta_{1}<\eta_{2}<\cdots<\eta_{m-2}<1,
\end{aligned}
$$

has been thoroughly studied by Gupta et al., see, for example, $[3,4,5]$.

The existence of positive solutions has been investigated by other authors. For example, Ma [11] has studied the second set of boundary conditions when all the $\alpha_{i}$ are nonnegative and $\sum_{i=1}^{m-2} \alpha_{i} \eta_{i}<1$ under the assumption that $f$ is either subor superlinear. 
More general boundary conditions have been studied by Ma and Castaneda [12], again when $f$ is either sub- or superlinear.

The special case of the 3-point BVP has been studied in greater detail, one reason being that the $m$-point BVP can be reduced to a 3-point BVP when all the coefficients $\alpha_{i}$ are positive [3,7]. The existence of a positive solution for the 3-point version of (1.2b) was established by Ma [10] under the condition $0<\alpha \eta<1$ for $f$ either sub- or superlinear. Under weaker conditions on $f$, He and Ge [6] showed the existence of three (and multiple) nonnegative solutions for the 3-point version of (1.2b) when $0<\alpha \eta<1$ while Webb [14] studied the existence of multiple positive solutions when $0<\alpha<1$ for (1.2a) and $0<\alpha \eta<1$ for $(1.2 \mathrm{~b})$.

The usual approach has been to write the BVP as an equivalent Hammerstein integral equation

$$
u(t)=\int_{0}^{1} k(t, s) g(s) f(u(s)) d s=: T u(t)
$$

and find a solution as a fixed point of the operator $T$ by using the classical theory of fixed-point index in cones. A different method is employed by Palamides [13], which also allows $f$ to depend on first-order derivatives and has a more general boundary condition at 0 , namely, $a u(0)-b u^{\prime}(0)=0$.

In the present paper, we want to show that requiring all the $\alpha_{i}$ to be nonnegative is much too restrictive, and that positive solutions exist more generally for both sets of boundary conditions. As in [14], our results allow more general behaviour on $f$ than being either sub- or superlinear.

In order to keep the calculations at a reasonable level, we concentrate on the 4-point BVPs. We suppose $\eta_{1}, \eta_{2}$ are given and we determine in each case necessary conditions on the parameters $\alpha_{1}, \alpha_{2}$ so that the kernel $k(t, s) \geq 0$ for all $0 \leq t, s \leq 1$. This determines a region in the $\left(\alpha_{1}, \alpha_{2}\right)$ plane which is unbounded and is much larger than the triangle

$$
\alpha_{1} \geq 0, \quad \alpha_{2} \geq 0, \quad \alpha_{1} \eta_{1}+\alpha_{2} \eta_{2}<1,
$$

which has been previously used for the BVP (1.2b).

We then show that if the parameters lie strictly inside these regions, then one or multiple positive solutions exist under suitable conditions on $f$. Our method utilises some known results of Lan [8] for the Hammerstein integral equation.

\section{Positive solutions of some Hammerstein integral equations}

We begin by recalling some results for the following Hammerstein integral equation:

$$
u(t)=\int_{0}^{1} k(t, s) g(s) f(u(s)) d s \equiv T u(t) .
$$


Although it is possible to give more general results (e.g., it is possible to replace $g(s) f(u(s))$ by $f(s, u(s))$ which satisfies Carathéodory conditions, and we can treat some discontinuous kernels $k$ ), for simplicity in the sequel, we will make the following assumptions on $f, g$, and the kernel $k$ :

$\left(\mathrm{C}_{1}\right) k:[0,1] \times[0,1] \rightarrow[0, \infty)$ is continuous;

$\left(\mathrm{C}_{2}\right) f: \mathbb{R} \rightarrow[0, \infty)$ is continuous;

$\left(\mathrm{C}_{3}\right) g \in L^{1}(0,1)$ and $g(s) \geq 0$ a.e.;

$\left(\mathrm{C}_{4}\right)$ there exist a measurable function $\Phi:[0,1] \rightarrow[0, \infty)$, a subinterval $[a, b]$ on which $\int_{a}^{b} g(s) d s>0$, and $c \in(0,1]$ such that

$$
\begin{array}{ll}
k(t, s) \leq \Phi(s) & \text { for } t, s \in[0,1], \\
k(t, s) \geq c \Phi(s) & \text { for } t \in[a, b], s \in[0,1] .
\end{array}
$$

This allows us to use the following cone $K$, of a type due to Guo (see, e.g., [2]), which is a subset of the cone $P$ of positive functions:

$$
K=\{u \in C[0,1]: u \geq 0, \min \{u(t): a \leq t \leq b\} \geq c\|u\|\} .
$$

Lemma 2.1 (see $[8,9])$. Under the above hypotheses, the map T defined in (2.1) maps $K$ into $K$ and is compact.

Definition 2.2. We define the following numbers:

$$
\begin{aligned}
m & =\left(\max _{t \in[0,1]} \int_{0}^{1} k(t, s) g(s) d s\right)^{-1}, \quad M=\left(\min _{t \in[a, b]} \int_{a}^{b} k(t, s) g(s) d s\right)^{-1}, \\
f^{0, \rho} & =\max _{u \in[0, \rho]} \frac{f(u)}{\rho}, \quad f^{0}=\limsup _{u \rightarrow 0^{+}} \frac{f(u)}{u}, \quad f^{\infty}=\limsup _{u \rightarrow+\infty} \frac{f(u)}{u}, \\
f_{c \rho, \rho} & =\min _{u \in[c \rho, \rho]} \frac{f(u)}{\rho}, \quad f_{0}=\liminf _{u \rightarrow 0^{+}} \frac{f(u)}{u}, \quad f_{\infty}=\liminf _{u \rightarrow+\infty} \frac{f(u)}{u} .
\end{aligned}
$$

This notation allows us to state the following theorem, a special case of some results from [8] proved by using the theory of fixed-point index.

Theorem 2.3. If $\left(C_{1}\right),\left(C_{2}\right),\left(C_{3}\right)$, and $\left(C_{4}\right)$ hold, then (2.1) has a positive solution in $K$ if one of the following conditions holds:

$\left(\mathrm{h}_{1}\right) 0 \leq f^{0}<m$ and $M<f_{\infty} \leq \infty$;

$\left(\mathrm{h}_{2}\right) M<f_{0} \leq \infty$ and $0 \leq f^{\infty}<m$.

Equation (2.1) has two positive solutions in $K$ if there is $\rho>0$ such that either of the following conditions holds:

$\left(\mathrm{S}_{1}\right) 0 \leq f^{0}<m, f_{c \rho, \rho}>c M$, and $0 \leq f^{\infty}<m$;

$\left(\mathrm{S}_{2}\right) M<f_{0} \leq \infty, f^{0, \rho}<m$, and $M<f_{\infty} \leq \infty$.

Under the hypothesis $\left(S_{1}\right)$, there are in fact 3 nonnegative solutions, but the third may be 0 . This result is similar to the result of [6], but the constant $m$ here is better (larger) than the constant used in [6]. 


\section{Positive solutions of the 4-point BVP (1.2a)}

We now consider the two 4-point BVPs in detail. We first consider the BVP

$$
u^{\prime \prime}(t)+g(t) f(u(t))=0, \quad \text { a.e on }[0,1]
$$

with boundary conditions

$$
u^{\prime}(0)=0, \quad u(1)=\alpha_{1} u\left(\eta_{1}\right)+\alpha_{2} u\left(\eta_{2}\right)
$$

If we write $\gamma_{1}=1-\alpha_{1}-\alpha_{2}$, then the solution of $u^{\prime \prime}=-y$ subject to the BCs (3.2) is

$$
\begin{aligned}
u(t)= & \frac{1}{\gamma_{1}}\left[\int_{0}^{1}(1-s) y(s) d s-\alpha_{1} \int_{0}^{\eta_{1}}\left(\eta_{1}-s\right) y(s) d s-\alpha_{2} \int_{0}^{\eta_{2}}\left(\eta_{2}-s\right) y(s) d s\right] \\
& -\int_{0}^{t}(t-s) y(s) d s .
\end{aligned}
$$

Thus the kernel (Green's function) is

$$
\begin{aligned}
k(t, s)= & \frac{1}{\gamma_{1}}(1-s) \\
& -\frac{\alpha_{1}}{\gamma_{1}}\left\{\begin{array}{ll}
\eta_{1}-s, & s \leq \eta_{1}, \\
0, & s>\eta_{1},
\end{array}-\frac{\alpha_{2}}{\gamma_{1}}\left\{\begin{array}{ll}
\eta_{2}-s, & s \leq \eta_{2}, \\
0, & s>\eta_{2},
\end{array}- \begin{cases}t-s, & s \leq t, \\
0, & s>t .\end{cases} \right.\right.
\end{aligned}
$$

For existence of positive solutions of (2.1), the standard assumption made is that $k(t, s) \geq 0$ for all $t, s$. If, for example, $k\left(t_{0}, s\right)<0$ for $s$ in some interval, then even the linear problem with a positive right-hand side can have a solution with $u\left(t_{0}\right)<0$. Hence we will investigate when $k(t, s) \geq 0$ for all $t, s$. This will determine a region in the $\left(\alpha_{1}, \alpha_{2}\right)$ plane and we will show that if $\left(\alpha_{1}, \alpha_{2}\right)$ lies in the interior of this region, then the hypothesis $\left(\mathrm{C}_{4}\right)$ is also satisfied, and hence positive solutions for the nonlinear problem can be shown to exist.

The requirement $k(t, s) \geq 0$ for all $t, s$ needs $\gamma_{1}>0$, that is, $\alpha_{1}+\alpha_{2}<1$ plus some other conditions which we explore now.

For a given $s, t \rightarrow k(t, s)$ is a decreasing function of $t$, so we investigate when $k(1, s) \geq 0$ for each $s$.

For $\eta_{2}<s<1$

$$
k(1, s)=\frac{1-s}{\gamma_{1}}-(1-s)=\frac{\left(\alpha_{1}+\alpha_{2}\right)}{\gamma_{1}}(1-s) .
$$

For $\eta_{1}<s \leq \eta_{2}$

$$
k(1, s)=\frac{1}{\gamma_{1}}\left[\left(\alpha_{1}+\alpha_{2}\right)(1-s)-\alpha_{2}\left(\eta_{2}-s\right)\right]
$$




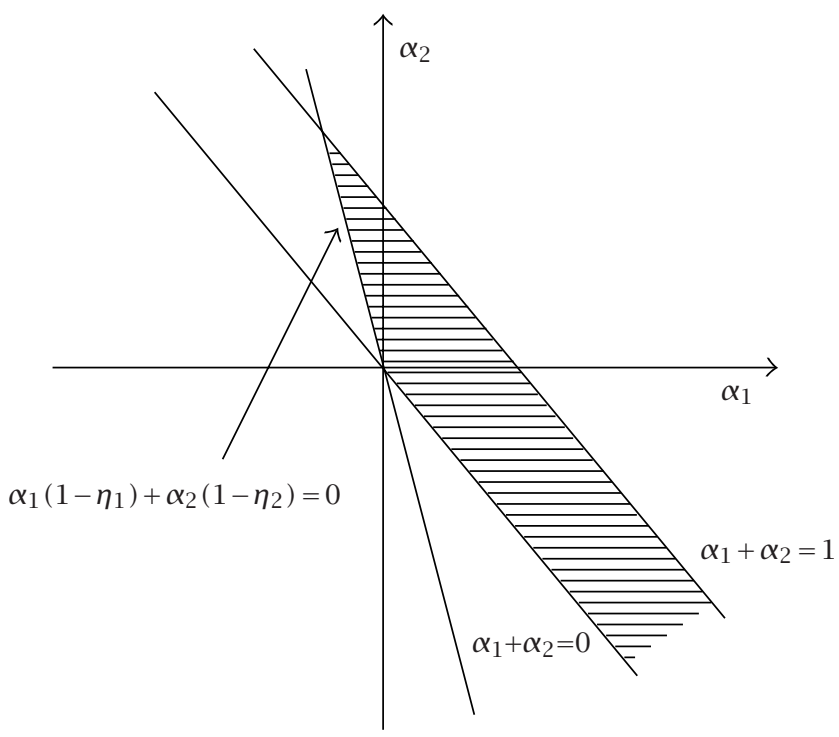

Figure 3.1. Region where the kernel is positive.

For $0<s \leq \eta_{1}$,

$$
k(1, s)=\frac{1}{\gamma_{1}}\left[\alpha_{1}\left(1-\eta_{1}\right)+\alpha_{2}\left(1-\eta_{2}\right)\right]
$$

Thus we need $\alpha_{1}+\alpha_{2}=1-\gamma_{1} \geq 0$ and $d_{1}:=\alpha_{1}\left(1-\eta_{1}\right)+\alpha_{2}\left(1-\eta_{2}\right) \geq 0$. These also ensure $\left(\alpha_{1}+\alpha_{2}\right)(1-s)-\alpha_{2}\left(\eta_{2}-s\right) \geq 0$ for $\eta_{1} \leq s \leq \eta_{2}$.

The region of the $\left(\alpha_{1}, \alpha_{2}\right)$ plane for which $k(t, s) \geq 0$ is therefore as shown in Figure 3.1.

Remark 3.1. We obtain the "region" for the 3-point BVP as the projection on the line $\alpha_{1}=0$, which gives the known condition $0<\alpha<1$, see [14].

We now show that if $\left(\alpha_{1}, \alpha_{2}\right)$ lies in the interior of the region of Figure 3.1 then the kernel satisfies $\left(\mathrm{C}_{4}\right)$, that is, suitable $\Phi$, subinterval $[a, b]$, and $c$ exist.

Upper bounds. For each $s$, the maximum of $k(t, s)$ occurs when $t=0$. So we may take $\Phi(s)=k(0, s)$.

Hence we have

$$
\Phi(s)= \begin{cases}\frac{1}{\gamma_{1}}(1-s), & \eta_{2}<s \leq 1, \\ \frac{1}{\gamma_{1}}\left(1-s-\alpha_{2}\left(\eta_{2}-s\right)\right), & \eta_{1}<s \leq \eta_{2}, \\ \frac{1}{\gamma_{1}}\left(d_{2}-\left(1-\alpha_{1}-\alpha_{2}\right) s\right), & 0<s \leq \eta_{1},\end{cases}
$$


where $d_{2}:=1-\alpha_{1} \eta_{1}-\alpha_{2} \eta_{2}$ and $d_{2}>1-\alpha_{1}-\alpha_{2}>0$ inside the region where $k \geq 0$.

Lower bounds on $[a, b]$. We take $[a, b]=\left[0, \eta_{1}\right]$. The minimum of $k(t, s)$ for $s$ fixed is $k\left(\eta_{1}, s\right)$. Hence we want to determine $c$ as large as possible so that $k\left(\eta_{1}, s\right) \geq c \Phi(s)$.

For $\eta_{2}<s<1$,

$$
k\left(\eta_{1}, s\right)=\frac{1}{\gamma_{1}}(1-s) .
$$

For $\eta_{1}<s \leq \eta_{2}$,

$$
k\left(\eta_{1}, s\right)=\frac{1}{\gamma_{1}}\left[1-s-\alpha_{2}\left(\eta_{2}-s\right)\right] .
$$

For $0<s \leq \eta_{1}$, letting

$$
d_{3}:=1-\eta_{1}-\alpha_{2}\left(\eta_{2}-\eta_{1}\right)=d_{1}+\left(1-\eta_{1}\right) \gamma_{1}>0,
$$

we have

$$
k\left(\eta_{1}, s\right)=\frac{1}{\gamma_{1}}\left[1-\eta_{1}-\alpha_{2}\left(\eta_{2}-\eta_{1}\right)\right]=\frac{1}{\gamma_{1}} d_{3} .
$$

So, $k_{\min } \geq c \Phi(s)$ for $t \in[a, b]$ if

$$
\frac{1}{\gamma_{1}} d_{3} \geq c \frac{1}{\gamma_{1}}\left[d_{2}-\left(1-\alpha_{1}-\alpha_{2}\right) s\right]
$$

for $0<s \leq \eta_{1}$. Thus $c=d_{3} / d_{2}$.

The constants $m, M$ from Definition 2.2 can be calculated for an explicitly given $g$. We give the results for the special case $g(s) \equiv 1$ as follows:

$$
\begin{aligned}
\frac{1}{m} & =\max _{t \in[0,1]} \int_{0}^{1} k(t, s) d s=\int_{0}^{1} k(0, s) d s=\frac{1}{2 \gamma_{1}}\left(1-\alpha_{1} \eta_{1}^{2}-\alpha_{2} \eta_{2}^{2}\right) \\
\frac{1}{M} & =\min _{t \in\left[0, \eta_{1}\right]} \int_{0}^{\eta_{1}} k(t, s) d s=\int_{0}^{\eta_{1}} k\left(\eta_{1}, s\right) d s \\
& =\frac{1}{\gamma_{1}} \eta_{1}\left(1-\eta_{1}-\alpha_{2}\left(\eta_{2}-\eta_{1}\right)\right)=\frac{1}{\gamma_{1}} \eta_{1} d_{3} .
\end{aligned}
$$

This gives the following result.

Theorem 3.2. Let $g(s) \equiv 1, c=d_{3} / d_{2}$, and let $m, M$ be as given in (3.14). Then, for $\left(\alpha_{1}, \alpha_{2}\right)$ in the interior of the region of Figure 3.1, the BVP (3.1), (3.2) has at least one positive solution if either $\left(h_{1}\right)$ or $\left(h_{2}\right)$ of Theorem 2.3 holds, and has two positive solutions if there is $\rho>0$ such that either $\left(S_{1}\right)$ or $\left(S_{2}\right)$ of Theorem 2.3 holds. 


\section{Positive solutions of the 4-point BVP (1.2b)}

We now study the BVP

$$
u^{\prime \prime}(t)+g(t) f(u(t))=0 \quad \text { a.e on }[0,1],
$$

with boundary conditions

$$
u(0)=0, \quad u(1)=\alpha_{1} u\left(\eta_{1}\right)+\alpha_{2} u\left(\eta_{2}\right) .
$$

For the BCs (4.2), if we set $\gamma=1-\alpha_{1} \eta_{1}-\alpha_{2} \eta_{2}>0$, the kernel is

$$
\begin{aligned}
k(t, s)= & \frac{t}{\gamma}(1-s) \\
& -\frac{\alpha_{1} t}{\gamma}\left\{\begin{array}{ll}
\eta_{1}-s, & s \leq \eta_{1} \\
0, & s>\eta_{1}
\end{array}-\frac{\alpha_{2} t}{\gamma}\left\{\begin{array}{ll}
\eta_{2}-s, & s \leq \eta_{2} \\
0, & s>\eta_{2}
\end{array}- \begin{cases}t-s, & s \leq t, \\
0, & s>t .\end{cases} \right.\right.
\end{aligned}
$$

We will show that $k(t, s) \geq 0$ for all $t, s$ if $0<\gamma \leq 1$ and

$$
d_{1}:=\alpha_{1}\left(1-\eta_{1}\right)+\alpha_{2}\left(1-\eta_{2}\right) \geq 0
$$

For $s>\eta_{2}$ and $t<s$,

$$
k(t, s)=\frac{1}{\gamma} t(1-s) \geq 0 .
$$

For $s>\eta_{2}$ and $t \geq s$,

$$
k(t, s)=\frac{1}{\gamma}[t(1-s)-\gamma(t-s)]=\frac{1}{\gamma}[t(1-\gamma-s)+\gamma s]
$$

and $k(t, s) \geq 0$ since $k(s, s)$ and $k(1, s)$ are both positive.

For $\eta_{1}<s \leq \eta_{2}$ and $t<s$,

$$
k(t, s)=\frac{1}{\gamma}\left[t(1-s)-\alpha_{2} t\left(\eta_{2}-s\right)\right]=\frac{t}{\gamma}\left[1-s-\alpha_{2}\left(\eta_{2}-s\right)\right] .
$$

Now

$$
1-s-\alpha_{2}\left(\eta_{2}-s\right) \geq \min \left\{1-\eta_{2}, 1-\eta_{1}-\alpha_{2}\left(\eta_{2}-\eta_{1}\right)\right\},
$$

where

$$
d_{4}:=1-\eta_{1}-\alpha_{2}\left(\eta_{2}-\eta_{1}\right)=\gamma\left(1-\eta_{1}\right)+d_{1} \eta_{1} \geq 0 .
$$

Note that $d_{4}=d_{3}$, but we have different hypotheses from the previous BC, so the positivity of $d_{4}$ has to be shown. Then we have

$$
k(t, s) \geq \frac{t}{\gamma} \min \left\{1-\eta_{2}, d_{4}\right\} \geq 0
$$


For $\eta_{1}<s \leq \eta_{2}$ and $t \geq s$, the minimum occurs either when $t=s$ or when $t=1$, so

$$
k(t, s) \geq \min \left\{\frac{1}{\gamma} s\left[1-s-\alpha_{2}\left(\eta_{2}-s\right)\right], \frac{1}{\gamma}\left[(1-\gamma)(1-s)-\alpha_{2}\left(\eta_{2}-s\right)\right]\right\} .
$$

Here

$$
\begin{gathered}
s\left[(1-s)-\alpha_{2}\left(\eta_{2}-s\right)\right] \geq \min \left\{\eta_{2}\left(1-\eta_{2}\right), \eta_{1} d_{4}\right\} \geq 0, \\
(1-\gamma)(1-s)-\alpha_{2}\left(\eta_{2}-s\right) \geq \min \left\{(1-\gamma)\left(1-\eta_{2}\right), d_{1}\right\} \geq 0 .
\end{gathered}
$$

For $0 \leq s \leq \eta_{1}$ and $t<s$,

$$
k(t, s)=\frac{1}{\gamma} t\left[\gamma-s\left(1-\alpha_{1}-\alpha_{2}\right)\right] \geq 0
$$

since $\gamma-\eta_{1}\left(1-\alpha_{1}-\alpha_{2}\right)=d_{4} \geq 0$.

For $0 \leq s \leq \eta_{1}$ and $t \geq s$, the minimum occurs either when $t=s$ or when $t=1$, and we have

$$
\begin{gathered}
k(1, s)=\frac{1}{\gamma}\left[\gamma s-s\left(1-\alpha_{1}-\alpha_{2}\right)\right]=\frac{1}{\gamma} s\left(d_{1}\right) \geq 0, \\
k(s, s)=\frac{1}{\gamma}\left[\gamma s-s^{2}\left(1-\alpha_{1}-\alpha_{2}\right)\right] \geq 0
\end{gathered}
$$

since it is equal to 0 when $s=0$, and when $s=\eta_{1}$,

$$
k\left(\eta_{1}, \eta_{1}\right)=\frac{\eta_{1}}{\gamma}\left[\gamma-\eta_{1}\left(1-\alpha_{1}-\alpha_{2}\right)\right]=\frac{1}{\gamma} d_{4}>0 .
$$

The region of the $\left(\alpha_{1}, \alpha_{2}\right)$ plane for which $k(t, s) \geq 0$ is therefore as shown in Figure 4.1, which is clearly much larger than the triangle in the first quadrant which is essentially the region previously used by other authors.

Remark 4.1. Projecting onto the line $\alpha_{1}=0$ gives the "region" for the 3-point BVP, $0<\alpha \eta<1$.

We now determine $\Phi$ and show that we may take $[a, b]=\left[\eta_{2}, 1\right]$.

Upper bounds. Since $k(0, s)=0$ and $t \mapsto k(t, s)$ is linear, with a jump in the gradient at $t=s$, the maximum occurs either when $t=s$ or when $t=1$.

For $s>\eta_{2}$ and $t<s$,

$$
k(t, s)=\frac{1}{\gamma} t(1-s) \leq \frac{1-s}{\gamma}
$$

For $s>\eta_{2}$ and $t \geq s$,

$$
k(t, s)=\frac{1}{\gamma}[t(1-s)-\gamma(t-s)]=\frac{1}{\gamma} t[(1-\gamma)-s]+s .
$$




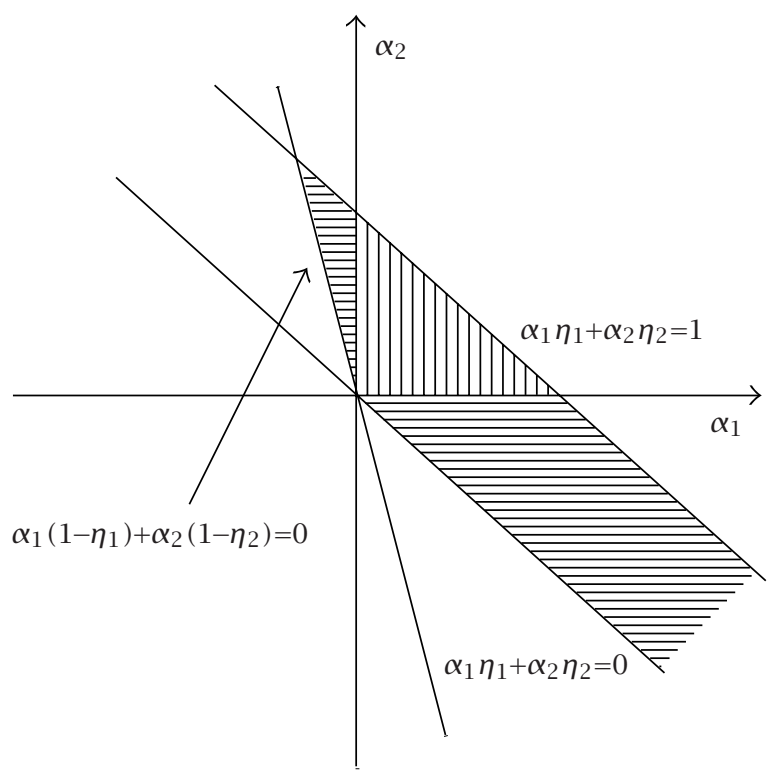

Figure 4.1. Region for positive kernel.

Here $k(s, s)=(1 / \gamma) s(1-s) \leq(1 / \gamma)(1-s)$ and

$$
k(1, s)=\frac{(1-\gamma)}{\gamma}(1-s) \leq \frac{1-s}{\gamma} .
$$

For $\eta_{1}<s \leq \eta_{2}$,

$$
\begin{aligned}
k(s, s) & =\frac{s}{\gamma}\left[1-s-\alpha_{2}\left(\eta_{2}-s\right)\right], \\
k(1, s) & =\frac{1}{\gamma}\left[(1-s)-\alpha_{2}\left(\eta_{2}-s\right)\right]+s \\
& =\frac{1}{\gamma}\left[(1-\gamma)(1-s)-\alpha_{2}\left(\eta_{2}-s\right)\right] .
\end{aligned}
$$

For $0 \leq s \leq \eta_{1}$, the maximum is either

$$
k(s, s)=\frac{s}{\gamma}\left[\gamma-\left(1-\alpha_{1}-\alpha_{2}\right) s\right]
$$

or

$$
k(1, s)=\frac{1}{\gamma} s\left[\gamma-\left(1-\alpha_{1}-\alpha_{2}\right)\right]=\frac{1}{\gamma} s d_{1} .
$$


Hence we can take $\Phi(s)$ as follows:

$$
\Phi(s)= \begin{cases}\frac{1}{\gamma}(1-s), & \eta_{2}<s \leq 1, \\ \frac{1}{\gamma}\left(1-s-\alpha_{2}\left(\eta_{2}-s\right)\right), & \eta_{1}<s \leq \eta_{2}, \\ \frac{1}{\gamma} s\left(\gamma-\left(1-\alpha_{1}-\alpha_{2}\right) s\right), & 0 \leq s \leq \eta_{1}, \alpha_{1}+\alpha_{2} \leq 1, \\ \frac{1}{\gamma} s\left(\alpha_{1}\left(1-\eta_{1}\right)+\alpha_{2}\left(1-\eta_{2}\right)\right), & 0 \leq s \leq \eta_{1}, \alpha_{1}+\alpha_{2}>1 .\end{cases}
$$

Lower bounds on $[a, b]=\left[\eta_{2}, 1\right]$. For the subinterval $[a, b]$, we must have $a>0$, and, guided by our knowledge of the 3 -point BVP, we choose $[a, b]=\left[\eta_{2}, 1\right]$.

For $s>\eta_{2}$ and $\eta_{2} \leq t<s$,

$$
k(t, s)=\frac{1}{\gamma} t(1-s) \geq \frac{1}{\gamma} \eta_{2}(1-s) .
$$

For $s>\eta_{2}$ and $t \geq s$,

$$
k(t, s)=\frac{1}{\gamma}[t(1-\gamma-s)+\gamma s],
$$

and the minimum occurs either at $t=s$ or at $t=1$ as follows:

$$
\begin{aligned}
k(s, s) & =\frac{1}{\gamma}[s(1-\gamma-s)+\gamma s] \\
& =\frac{1}{\gamma} s(1-s) \geq \frac{1}{\gamma} \eta_{2}(1-s), \\
k(1, s) & =\frac{1-\gamma}{\gamma}(1-s) .
\end{aligned}
$$

Hence, $k_{\min } \geq c \Phi(s)$ for $s>\eta_{2}$ if

$$
c \leq \min \left\{\eta_{2}, 1-\gamma\right\} .
$$

For $\eta_{2} \geq s>\eta_{1}$ and $t \in\left[\eta_{2}, 1\right]$,

$$
\begin{gathered}
k(t, s)=\frac{1}{\gamma}\left[t(1-s)-\alpha_{2}\left(\eta_{2}-s\right)-\gamma\right]+s, \\
k_{\min }=\min \left\{\frac{1}{\gamma} \eta_{2}\left(1-s-\alpha_{2}\left(\eta_{2}-s\right)\right)-\eta_{2}+s, \frac{1}{\gamma}\left(1-s-\alpha_{2}\left(\eta_{2}-s\right)\right)-1+s\right\} .
\end{gathered}
$$

We want $k_{\min } \geq c \Phi(s)$, where

$$
\Phi(s)=\frac{1}{\gamma}\left(1-s-\alpha_{2}\left(\eta_{2}-s\right)\right) \quad \text { for } \eta_{1}<s \leq \eta_{2} .
$$


This requires

$$
\begin{gathered}
\eta_{2} \Phi(s)-\eta_{2}+s \geq c \Phi(s), \\
\Phi(s)-1+s \geq c \Phi(s) .
\end{gathered}
$$

Condition (4.29) needs

$$
\eta_{2}-s \leq\left(\eta_{2}-c\right) \Phi(s) \text { for } \eta_{1}<s \leq \eta_{2} .
$$

This is satisfied if $c \leq \eta_{2}$ and

$$
\eta_{2}-\eta_{1} \leq\left(\eta_{2}-c\right) \Phi\left(\eta_{1}\right)=\left(\eta_{2}-c\right) \frac{d_{4}}{\gamma} .
$$

Note that we must have

$$
\eta_{2}-\frac{\left(\eta_{2}-\eta_{1}\right) \gamma}{d_{4}}>0
$$

for $c$ to exist. In fact, using (4.9), we have

$$
\eta_{2}-\frac{\left(\eta_{2}-\eta_{1}\right) \gamma}{d_{4}}>\eta_{2}-\frac{\left(\eta_{2}-\eta_{1}\right)}{1-\eta_{1}}=\frac{\eta_{1}\left(1-\eta_{2}\right)}{1-\eta_{1}}>0 .
$$

Hence we want

$$
c \leq \eta_{2}-\frac{\left(\eta_{2}-\eta_{1}\right) \gamma}{d_{4}}
$$

Condition (4.30) needs

$$
1-s \leq(1-c) \Phi(s) \text { for } \eta_{1}<s \leq \eta_{2} .
$$

When $s=\eta_{2},(4.36)$ is

$$
1-\eta_{2} \leq(1-c) \frac{\left(1-\eta_{2}\right)}{\gamma}
$$

so $c \leq 1-\gamma$ suffices.

When $s=\eta_{1},(4.36)$ is

$$
1-\eta_{1} \leq(1-c) \frac{d_{4}}{\gamma}
$$

Since $\eta_{1} d_{1}+\left(1-\eta_{1}\right) \gamma=d_{4}$ from (4.9), this yields

$$
c \leq 1-\frac{\left(1-\eta_{1}\right) \gamma}{d_{4}}=\eta_{1} \frac{d_{1}}{d_{4}} .
$$


For $0 \leq s \leq \eta_{1}$ and $t \geq \eta_{2}$,

$$
k(t, s)=\frac{1}{\gamma} t\left(\alpha_{1}+\alpha_{2}-1\right) s+s .
$$

If $\alpha_{1}+\alpha_{2}>1$, then $k(t, s)$ is increasing in $t$, so the minimum is at $t=\eta_{2}$ as follows:

$$
k_{\min }=\frac{1}{\gamma}\left[\eta_{2}\left(\alpha_{1}+\alpha_{2}-1\right) s+\gamma s\right]=\frac{1}{\gamma}\left(1-\eta_{2}+\alpha_{1}\left(\eta_{2}-\eta_{1}\right)\right) s .
$$

In order that $k_{\min } \geq c \Phi(s)$, we need

$$
c \leq \frac{\left(1-\eta_{2}+\alpha_{1}\left(\eta_{2}-\eta_{1}\right)\right)}{d_{1}} .
$$

Note that

$$
1-\eta_{2}+\alpha_{1} \eta_{2}-\alpha_{1} \eta_{1}=\gamma+\eta_{2}\left(\alpha_{1}+\alpha_{2}-1\right)>\gamma>0
$$

since this is the case $\alpha_{1}+\alpha_{2}>1$. Hence, for this case, we can take

$$
c \leq \frac{\gamma}{d_{1}} .
$$

When $\alpha_{1}+\alpha_{2} \leq 1, k(t, s)$ is decreasing in $t$, so

$$
k_{\min }=\frac{1}{\gamma}\left[\left(\alpha_{1}+\alpha_{2}-1\right) s+\gamma s\right]=\frac{s}{\gamma} d_{1} .
$$

We want

$$
\frac{s}{\gamma} d_{1} \geq c \frac{1}{\gamma} s\left(\gamma-\left(1-\alpha_{1}-\alpha_{2}\right) s\right)
$$

hence we want $c \leq d_{1} / d_{4}$. The total requirement is therefore

$$
c \leq \min \left\{1-\gamma, \eta_{1} \frac{d_{1}}{d_{4}}, \frac{\gamma}{d_{1}}, \eta_{2}-\frac{\left(\eta_{2}-\eta_{1}\right) \gamma}{d_{4}}\right\} .
$$

As all the requisite conditions have been now verified, we immediately have the following theorem.

Theorem 4.2. Let $[a, b]=\left[\eta_{2}, 1\right]$ and suppose that $\int_{\eta_{2}}^{1} g(s) d s>0$. Let $c$ satisfy (4.47) and let $m, M$ be as in Definition 2.2. Then, for $\left(\alpha_{1}, \alpha_{2}\right)$ in the interior of the region of Figure 4.1, the BVP (4.1), (4.2) has at least one positive solution if either $\left(h_{1}\right)$ or $\left(h_{2}\right)$ of Theorem 2.3 holds, and has two positive solutions if there is $\rho>0$ such that either $\left(S_{1}\right)$ or $\left(S_{2}\right)$ of Theorem 2.3 holds. 
In the special case when $g(s) \equiv 1, m, M$ are readily calculated. In fact,

$$
\begin{aligned}
\frac{1}{m} & =\max _{t \in[0,1]} \int_{0}^{1} k(t, s) d s \\
& =\max _{t \in[0,1]} \frac{1}{2 \gamma}\left[t\left(1-\alpha_{1} \eta_{1}^{2}-\alpha_{2} \eta_{2}^{2}\right)-\gamma t^{2}\right] \\
& =\frac{1}{8 \gamma^{2}}\left(1-\alpha_{1} \eta_{1}^{2}-\alpha_{2} \eta_{2}^{2}\right)^{2}, \\
\frac{1}{M} & =\min _{t \in\left[\eta_{2}, 1\right]} \int_{\eta_{2}}^{1} k(t, s) d s \\
& =\min _{t \in\left[\eta_{2}, 1\right]} \frac{1}{2 \gamma}\left[t\left(1-\eta_{2}\right)^{2}-\gamma\left(t-\eta_{2}\right)^{2}\right] \\
& =\min _{2}\left\{\eta_{2}, 1-\gamma\right\} \frac{\left(1-\eta_{2}\right)^{2}}{2 \gamma} .
\end{aligned}
$$

Conclusion. It is possible to extend our methods to deal with 5,6,..-point BVPs, but we feel this would be only worthwhile if required by an explicit application. Our aim is to show that the "obvious" extension of the condition of the 3-point BVP that requires positivity of the coefficients $\alpha_{i}$ is far from optimal.

\section{Acknowledgment}

This research was partially supported by a visiting professorship of GNAMPA.

\section{References}

[1] A. V. Bitsadze and A. A. Samarskii, On some simple generalizations of linear elliptic boundary problems, Soviet Math. Dokl. 10 (1969), no. 2, 398-400.

[2] D. Guo and V. Lakshmikantham, Nonlinear Problems in Abstract Cones, Notes and Reports in Mathematics in Science and Engineering, vol. 5, Academic Press, Massachusetts, 1988.

[3] C. P. Gupta, A generalized multi-point boundary value problem for second order ordinary differential equations, Appl. Math. Comput. 89 (1998), no. 1-3, 133-146.

[4] C. P. Gupta, S. K. Ntouyas, and P. Ch. Tsamatos, On an m-point boundary-value problem for second-order ordinary differential equations, Nonlinear Anal. 23 (1994), no. $11,1427-1436$.

[5] C. P. Gupta and S. I. Trofimchuk, Existence of a solution of a three-point boundary value problem and the spectral radius of a related linear operator, Nonlinear Anal. 34 (1998), no. 4, 489-507.

[6] X. He and W. Ge, Triple solutions for second-order three-point boundary value problems, J. Math. Anal. Appl. 268 (2002), no. 1, 256-265.

[7] V. A. Il'in and E. I. Moiseev, Nonlocal boundary value problem of the first kind for a Sturm-Liouville operator in its differential and finite difference aspects, Differ. Equ. 23 (1987), no. 7, 803-810.

[8] K. Q. Lan, Multiple positive solutions of semilinear differential equations with singularities, J. London Math. Soc. (2) 63 (2001), no. 3, 690-704. 
[9] K. Q. Lan and J. R. L. Webb, Positive solutions of semilinear differential equations with singularities, J. Differential Equations 148 (1998), no. 2, 407-421.

[10] R. Ma, Positive solutions of a nonlinear three-point boundary-value problem, Electron. J. Differential Equations 1999 (1999), no. 34, 1-8.

[11] _ Positive solutions of a nonlinear m-point boundary value problem, Comput. Math. Appl. 42 (2001), no. 6-7, 755-765.

[12] R. Ma and N. Castaneda, Existence of solutions of nonlinear m-point boundary-value problems, J. Math. Anal. Appl. 256 (2001), no. 2, 556-567.

[13] P. K. Palamides, Positive and monotone solutions of an m-point boundary-value problem, Electron. J. Differential Equations 2002 (2002), no. 18, 1-16.

[14] J. R. L. Webb, Positive solutions of some three point boundary value problems via fixed point index theory, Nonlinear Anal. 47 (2001), 4319-4332.

Gennaro Infante: Dipartimento di Matematica, Università della Calabria, 87036 Arcavacata di Rende, Cosenza, Italy

E-mail address: infanteg@unical.it

J. R. L. Webb: Department of Mathematics, University of Glasgow, Glasgow G12 8QW, UK

E-mail address: jrlw@maths.gla.ac.uk 


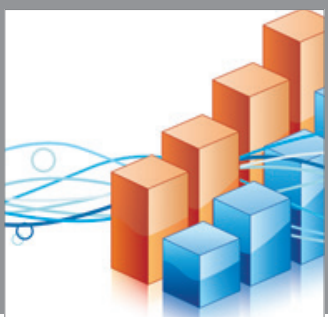

Advances in

Operations Research

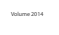

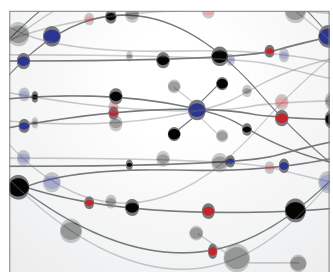

\section{The Scientific} World Journal
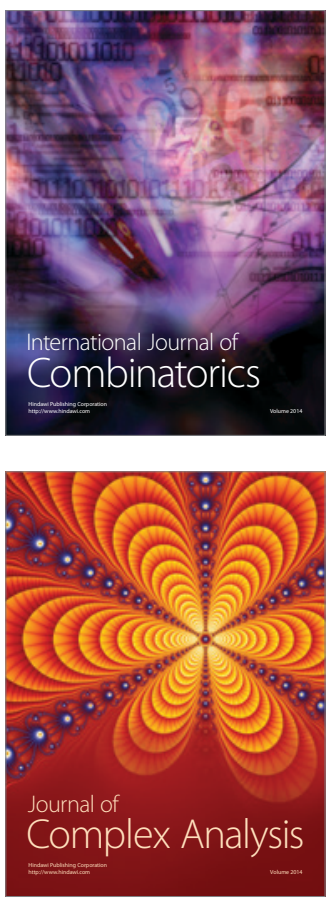

International Journal of

Mathematics and

Mathematical

Sciences
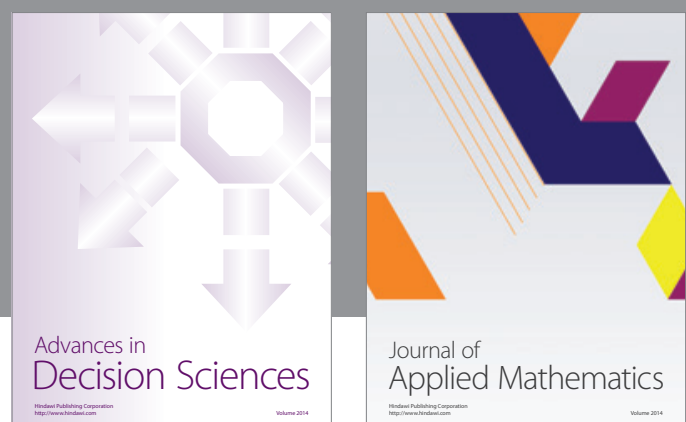

Journal of

Applied Mathematics
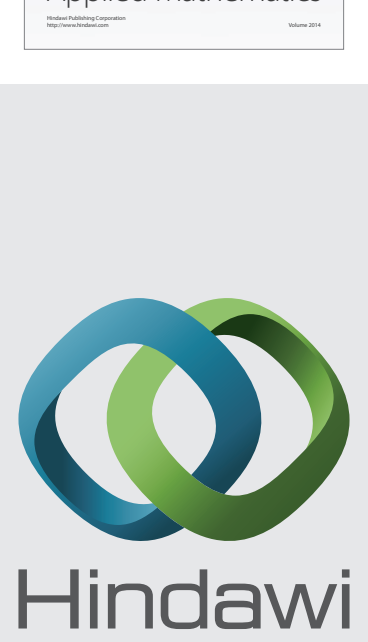

Submit your manuscripts at http://www.hindawi.com
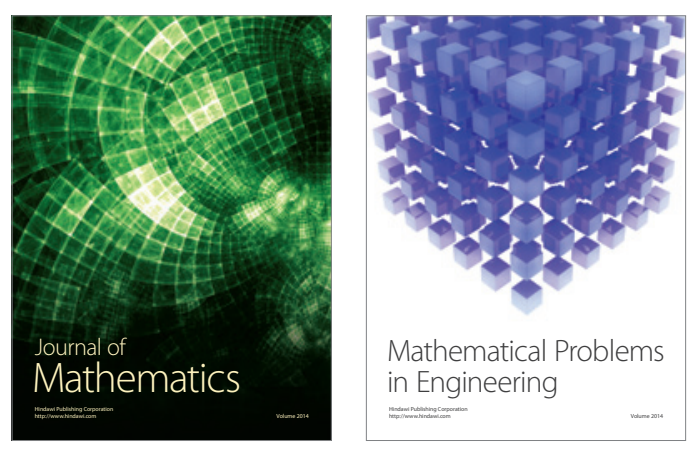

Mathematical Problems in Engineering
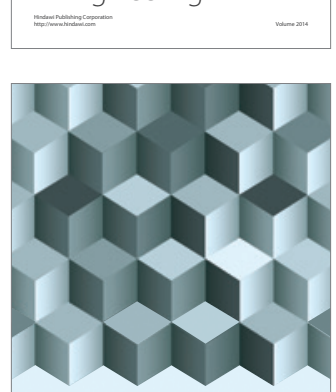

Journal of

Function Spaces
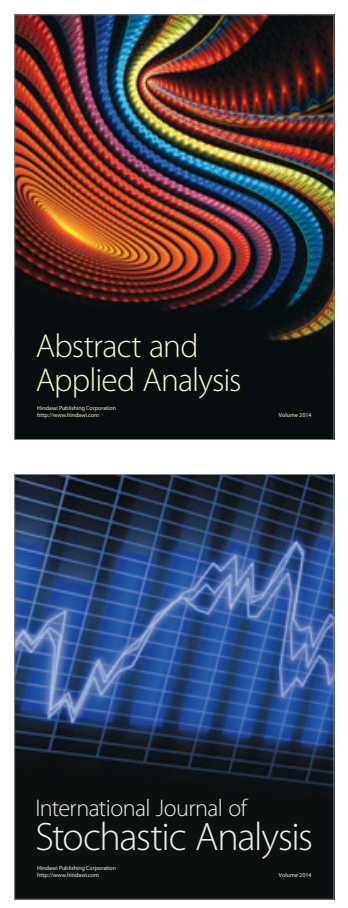

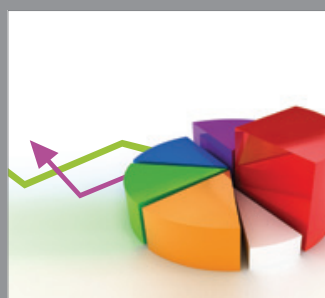

ournal of

Probability and Statistics

Promensencen
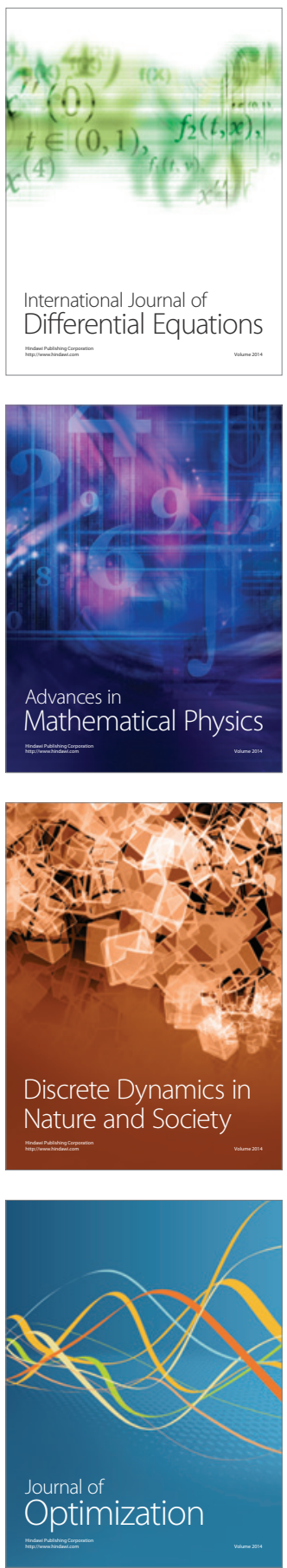